\title{
Declines in skeletal muscle quality vs. size following two weeks of knee joint immobilization
}

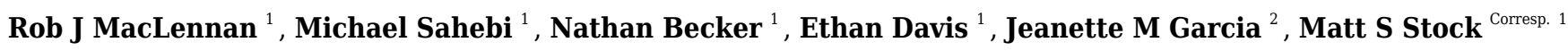 \\ ${ }^{1}$ School of Kinesiology and Physical Therapy, University of Central Florida, Orlando, Florida, United States of America \\ 2 Department of Health Sciences, University of Central Florida, Orlando, Florida, United States of America \\ Corresponding Author: Matt S Stock \\ Email address: matt.stock@ucf.edu
}

BACKGROUND. Disuse of a muscle group, which occurs during bedrest, spaceflight, and limb immobilization, results in atrophy. It is unclear, however, if the magnitude of decline in skeletal muscle quality is similar to that for muscle size. The purpose of this study was to examine the effects of two weeks of knee joint immobilization on vastus lateralis and rectus femoris echo intensity and cross-sectional area. METHODS. Thirteen females (mean \pm SD age $=21 \pm 2$ years) underwent two weeks of left knee joint immobilization via ambulating on crutches and use of a brace. B-mode ultrasonography was utilized to obtain transverse plane images of the immobilized and control vastus lateralis and rectus femoris at pretest and following immobilization. Effect size statistics and two-way repeated measures analyses of variance were used to interpret the data. RESULTS. No meaningful changes were demonstrated for the control limb and the rectus femoris of the immobilized limb. Analyses showed a large increase in vastus lateralis echo intensity (i.e., decreased muscle quality) for the immobilized $\operatorname{limb}(p=.006$, Cohen's $d=.918)$. For vastus lateralis cross-sectional area, no time $\times$ limb interaction was observed $(p=.103)$, but the effect size was moderate $(d=.570)$. There was a significant association between the increase in vastus lateralis echo intensity and the decrease in cross-sectional area $(r=-.649, p=$ .016). CONCLUSION. In female participants, two weeks of knee joint immobilization resulted in greater deterioration of muscle quality than muscle size. Echo intensity appears to be an attractive clinical tool for monitoring muscle quality during disuse. 
1 DECLINES IN SKELETAL MUSCLE QUALITY VS. SIZE 2 FOLLOWING TWO WEEKS OF KNEE JOINT 3 IMMOBILIZATION

4

5

6

7

8 9

21

22

23

24

25

26

27

28

29

30

31

32

33

34

35

36

37

38

39

40

41
Rob J. MacLennan ${ }^{1}$, Michael Sahebi ${ }^{1}$, Nathan Becker ${ }^{1}$, Ethan Davis ${ }^{1}$, Jeanette M. Garcia ${ }^{2}$, Matt S. Stock ${ }^{1}$

${ }^{1}$ School of Kinesiology and Physical Therapy, University of Central Florida, Orlando, Florida, USA

${ }^{2}$ Department of Health Sciences, University of Central Florida, Orlando, Florida, USA

Corresponding Author:

Matt S. Stock ${ }^{1}$

12805 Pegasus Drive, HPA 1 - Room 258, Orlando, FL 32816-2205, USA

Email address: matt.stock@ucf.edu 


\section{Abstract}

43 Background. Disuse of a muscle group, which occurs during bedrest, spaceflight, and limb

44

45

46

47

48

49

50

51

52

53

54

55

56

57

58

59

60

61

62

63

64

65

66

67

68

69

70

71

72

73

74

75

76

77

78

79

80

81

immobilization, results in atrophy. It is unclear, however, if the magnitude of decline in skeletal muscle quality is similar to that for muscle size. The purpose of this study was to examine the effects of two weeks of knee joint immobilization on vastus lateralis and rectus femoris echo intensity and cross-sectional area.

Methods. Thirteen females (mean $\pm \mathrm{SD}$ age $=21 \pm 2$ years) underwent two weeks of left knee joint immobilization via ambulating on crutches and use of a brace. B-mode ultrasonography was utilized to obtain transverse plane images of the immobilized and control vastus lateralis and rectus femoris at pretest and following immobilization. Effect size statistics and two-way repeated measures analyses of variance were used to interpret the data.

Results. No meaningful changes were demonstrated for the control limb and the rectus femoris of the immobilized limb. Analyses showed a large increase in vastus lateralis echo intensity (i.e., decreased muscle quality) for the immobilized $\operatorname{limb}(p=.006$, Cohen's $d=.918)$. For vastus lateralis cross-sectional area, no time $\times$ limb interaction was observed $(p=.103)$, but the effect size was moderate $(d=.570)$. There was a significant association between the increase in vastus lateralis echo intensity and the decrease in cross-sectional area $(r=-.649, p=.016)$.

Conclusion. In female participants, two weeks of knee joint immobilization resulted in greater deterioration of muscle quality than muscle size. Echo intensity appears to be an attractive clinical tool for monitoring muscle quality during disuse.

\section{Introduction}

Muscle unloading or disuse, such as what occurs during bed rest and removal of weight bearing conditions, results in muscular atrophy (Berg et al. 1997; Tesch et al. 2004; Wall et al. 2014). These changes present several significant challenges for health care providers, particularly following surgical procedures and bedrest in older adults, as muscle weakness is a strong predictor of mortality (Newman et al. 2006). Responses to disuse vary by sex (Deschenes et al. 2009) and muscle group (Schulze et al. 2002; Tesch et al. 2004), with greater declines in females and the knee joint, respectively. In athletics, females have a roughly three times greater incidence of anterior cruciate ligament (ACL) tears during soccer and basketball versus males, with many of these injuries occurring during non-contact situations (Prodromos et al. 2007; Renstrom et al. 2008). Female athletes that play sports year-round have an ACL tear rate of approximately 5\% (Renstrom et al. 2008). These issues are not limited to competitive athletes, however. ACL tears are common among any population that engages in physical activity and sports. It has been reported that the overall age- and sex-adjusted annual incidence of ACL tears among the general population was 68.6 per 100,000 person-years, with a substantial increase over time regardless of age (Sanders et al. 2016). Rehabilitation of the knee joint following surgical interventions has major social and economic consequences, with one study estimating a cost of $>\$ 2$ billion for an annual incidence of 200,000 ACL reconstructions in the U.S (Mather et al. 2013). Following ACL reconstruction surgery, specifically with a concomitant meniscal

Peer] reviewing PDF | (2019:08:40344:2:0:NEW 7 Nov 2019) 
82 repair, patients are often placed under temporary bed rest to maintain graft integrity, as well as

83 reduce compressive forces and pain at the knee joint (Hiemstra et al. 2009). Following short-term

84 immobilization, patients are typically administered a knee brace to be worn for all weight

85 bearing activities.

86 Given the implications for long-term health, changes in muscle size (i.e., hypertrophy and

87 atrophy) following various interventions have historically garnered substantial interest from

88 researchers and clinicians. In recent years, however, investigators have begun to consider

89 changes in muscle quality via analysis of echo intensity (Lopez et al. 2017). The premise behind

90 its use is that the degree of darkness/brightness of a given region of interest characterizes tissue

91 composition, as darker images (lower values) represent skeletal muscle tissue and brighter

92 images (higher values) represent non-contractile elements. While echo intensity's precise

93 underpinnings have yet to be directly verified, anatomical investigations carried out in humans

94 and animals have demonstrated that it strongly reflects fibrous tissue content (Arts et al. 2012;

95 Pillen et al. 2009), as well as intramuscular adiposity (Nishihara et al. 2014; Reimers et al. 1993;

96 Young et al. 2015). Echo intensity also seems to be at least moderately correlated with

97 performance measures such as muscle strength (Mota et al. 2017), stair-climbing ability

98 (Kleinberg et al. 2016), and chair stand ability (Rech et al. 2014; Lopez et al. 2017), and these

99 associations have been reported from adolescents (Stock et al. 2017) through older adults

100 (Watanabe et al. 2013). Furthermore, echo intensity can be easily analyzed with B-mode

101 ultrasonography. Though its image quality still lags behind CT and MRI, ultrasonography's

102 portability, cost, and time efficiency make it a realistic option for clinicians and practitioners

103 with limited resources. While more research and normative data are needed, at this point,

104 ultrasonography-based echo intensity shows promise as a tool for tracking changes in muscle

105 quality.

$106 \quad$ Understanding the neuromuscular and morphological adaptations that occur during short-

107 term disuse will allow for the development of more effective and timely preventative

108 interventions. While there is evidence that resistance training may affect echo intensity in older

109 people (Radaelli et al. 2014), evidence of this relationship in young people is less clear.

110 Furthermore, as additional imaging analytical techniques become available, it is important to

111 understand which variables may be more or less sensitive to changes in muscle morphology,

112 particularly in clinical settings. While the atrophic effects of limb immobilization have been well

113 established (Hackney and Ploutz-Snyder 2012), the extent to which muscle quality deteriorates is

114 unclear. Thus, the purpose of this study was to examine the effects of two weeks of knee joint

115 immobilization on vastus lateralis and rectus femoris muscle quality and size in college-aged

116 females. Based on previous studies (Deschenes et al. 2017; Hackney and Ploutz-Snyder 2012),

117 we hypothesized that skeletal muscle size would show a small-to-moderate decrease following

118 immobilization. In contrast, we speculated that muscle quality would deteriorate to a greater

119 extent, which would be suggestive of rapid intramuscular fat and/or fibrous tissue infiltration

120 during disuse, rather than reduction in muscle size.

121 
122 Materials \& Methods

123 Experimental Approach to the Problem

124 This investigation utilized a within-participants design in which B-mode ultrasonography

125 imaging was performed for the vastus lateralis and rectus femoris muscles of immobilized (left)

126 and control (right) limbs. All participants were right leg dominant. Data was collected during

127 baseline testing (PRE) and two weeks later (POST). All visits to the laboratory occurred at the

128 same time of day ( \pm one hour) throughout the course of the study. All imaging and image

129 analyses were performed by the same investigators utilizing methods described in our previous

130 publications (Burton and Stock 2018; Mota et al. 2017). Throughout the study, participants were

131 asked to refrain from alcohol, keep their dietary habits consistent, and comply with the

132 immobilization protocol. Participants kept a three-day food log, which was primarily meant to

133 serve as a reminder for the need for consistency of health behaviors throughout the study. Each

134 participant was assigned to a study investigator, who served as her direct point of contact and

135 was always available.

136 Participants

137 Due to the novelty of this topic, the ability to predict the observed effect size was limited.

138 As such, an a priori power analysis was not carried out as recommended by previous

139 investigators (Beck 2013). It should be noted, however, that a recent systematic review found

140 that lower limb immobilization studies have reported data for $\leq 13$ participants, with $\leq 10$ being

141 typical (Campbell et al. 2019). Thus, the final sample size described below is $\geq$ what has

142 previously been utilized in the relevant literature. This study's post-hoc power analyses for

143 particularly relevant outcomes have been included in our Supplemental Files.

144

Twenty-six healthy, college-aged females originally enrolled in this study. Recruitment

145 methods included the use of flyers, social media posts, marketing on laboratory and university websites, and presentations in group settings. Inclusion criteria included females between the ages of 18-35 years, a body mass index $\leq 30 \mathrm{~kg} / \mathrm{m}^{2}$, and willingness to comply with the study's demands. Major exclusion criteria included the following nine conditions: 1) personal or family history of blood clots; 2) neuromuscular or metabolic disease; 3 ) osteoarthritis; 4) previous surgery on the hip or knee joints; 5) use of an assistive walking device within the previous year; 6) myocardial infarction within the past year; 7) pregnancy; 8) use of contraceptives within the previous 90 days (Vinogradova et al. 2015); 9) musculoskeletal pain or discomfort in any of the major joints. Recent lower-body resistance or aerobic training participation was not considered exclusionary, trained and untrained individuals experience similar relative declines in lean mass following knee joint immobilization (Deschenes et al. 2017). Participants completed an in-house informed consent form. The study protocol was approved by the University of Central Florida's Institutional Review Board (Study \# BIO-17-13642). Participants were compensated $\$ 350$ for completing the study. 
161

162

163

164

165

166

167

168

169

170

171

172

173

174

175

176

177

178

179

180

181

182

183

184

185

186

187

188

189

190

191

192

193

194

195

196

197

198

199

200

Out of the 26 participants that enrolled, eleven withdrew. Reasons for withdrawing included, but were not limited to, time constraints, changes in contraceptive use, difficulty accomplishing daily activities and subsequent inability to comply with the study protocol, and discomfort. Out of the fifteen participants that completed the study, two were removed from this paper's statistical analysis, one because of difficulty with image analysis and another because of accelerometer data demonstrating non-compliance (details described below). Therefore, 13 participants (mean $\pm \mathrm{SD}$ age $=21 \pm 2$ years, mass $=61.6 \pm 4.6 \mathrm{~kg}$, height $=164.7 \pm 6.1 \mathrm{~cm})$ were included in our final data set.

\section{Immobilization Procedures}

During the pretesting data collection session, participants were properly fit for the use of a knee joint immobilization brace (T Scope ${ }^{\circledR}$ Premier Post-Op Knee Brace, Breg, Inc., Carlsbad, CA, USA). Each brace was locked at a $90^{\circ}$ angle, which allowed the left knee extensors to remain relaxed and unloaded while preventing the toes from contacting the ground. A $90^{\circ} \mathrm{knee}$ joint angle was a conservative approach to ensuring that the toes of the left foot did not come into contact with the ground while ambulating on the crutches. Participants wore the brace at all times, except during periods of sleep. Participants were also asked to keep their left leg in the brace and covered with a plastic bag when showering. To ensure safety and comfort while showering, each participant was offered a shower chair (Medline Shower Chair Bath Seat with Padded Armrests and Back, Medline Industries, Inc., Northfield, IL, USA). Participants were also provided and properly fit for axillary crutches (Cardinal Health Axillary Crutch, Adult, Height 62-70 in, Adjustable, Cardinal Health, Inc., Dublin, OH, USA) using guidelines described by Fairchild (2012). Training was provided on proper use of crutches in navigating the community including curbs, stairs, and sidewalks (Figure 1). Participants were advised that they would likely have trouble with activities of daily living such as transportation and cleaning, to allow additional time to complete tasks, and to plan ahead in order to avoid unsafe conditions (e.g., ensure a towel was near the shower and to sufficiently dry off). In addition to use of the brace and crutches, participants were urged to refrain from weight bearing.

\section{[FIGURE 1 ABOUT HERE]}

Each participant wore a stocking (Rolyan Extra Soft Stockinette, 100\% Cotton, Performance Health, Warrenville, IL, USA) underneath their brace that spanned the length of the left lower extremity from the proximal thigh to the ankle. This stocking was worn to improve comfort while wearing the knee brace and to minimize the risk of adverse skin reactions. The stocking could be taken off before bed but was worn during all daytime activities. To reduce the risk of blood clots, participants were also given nighttime compression stockings (Medi-Pak Anti-Embolism Stockings, McKesson, San Francisco, CA, USA) to be worn over the left leg while they slept. Basic hygiene was discussed with the participants, and they were instructed to notify the researchers immediately in the event that they noticed any irritation, redness, or swelling; this was not reported by any of the participants.

Consistent with previous knee joint immobilization studies (Deschenes et al. 2008; Deschenes et al. 2009), each participant performed light range of motion movements at the ankle 
201

202

203

204

205

206

207

208

209

210

211

212

213

214

215

216

217

218

219

220

221

222

223

224

225

226

227

228

229

230

231

232

233

234

235

236

237

238

239

and knee while lying supine in bed. These activities were performed twice daily (morning and evening) in an effort to minimize the risk of vascular pathology and muscular contractures. A video containing instructions was provided to participants, as was as a handout with detailed directions of performance of the exercises. The compressive stocking was worn during the exercise. Finally, to further ensure compliance and safety, study investigators text messaged or spoke with the participants via telephone daily.

\section{Ultrasonography Measurements and Analysis}

Ultrasonography images for the vastus lateralis and rectus femoris muscles of both limbs were taken with a portable B-mode imaging device (GE Logiq e BT12, GE Healthcare, Milwaukee, WI, USA) and a multi-frequency linear-array probe (12 L-RS, 5-13 MHz, 38.4-mm field of view; GE Healthcare, Milwaukee, WI, USA). The panoramic function (LogiqView, GE Healthcare, Milwaukee, WI, USA) was used to obtain images of the vastus lateralis and rectus femoris in the transverse plane. Prior to ultrasound measurements, subjects laid in the supine position for 5 minutes in order to allow the redistribution of fluids from their quadriceps muscles. The images were taken at $50 \%$ of the length of the femur. A high-density foam pad was secured around the thigh with an adjustable strap to ensure probe movement in the transverse plane. Ultrasonography settings (Frequency: $10 \mathrm{MHz}$, Gain: 55dB, Dynamic Range: 72) were kept consistent across participants. To ensure optimal image clarity, a standardized depth of $5.0 \mathrm{~cm}$ was utilized. However, for three participants, a greater depth was necessary to adequately capture each muscle belly. For these three participants, depths of 6.0, 6.0, and $7.0 \mathrm{~cm}$ were utilized. Depth for each participant was kept consistent across trials. A generous amount of water-soluble transmission gel (Aquasonic 100 ultrasonography transmission gel, Parker Laboratories, Inc., Fairfield, NJ, USA) was applied to the skin to allow immersion of the probe surface during measurement to enhance acoustic coupling. Three images of each muscle were obtained, and mean values were used for statistical analyses.

The ultrasonography images were digitized and examined with ImageJ software (version 1.46, National Institutes of Health, Bethesda, MD, USA) at the conclusion of the study. The polygon function was used to outline the borders of the vastus lateralis and rectus femoris. After scaling the image units from pixels to $\mathrm{cm}$, muscle cross-sectional area $\left(\mathrm{cm}^{2}\right)$ was determined with the polygon function. Echo intensity was also assessed by computer-aided gray-scale analysis using the histogram function. The echo intensity values were determined as the corresponding index of muscle quality ranging between 0 and 255 arbitrary units (AU). Recently, Young et al. (2015) provided an equation that allows investigators to correct rectus femoris echo intensity for the magnitude of subcutaneous thickness over the muscle (corrected echo intensity $=$ raw echo intensity + [subcutaneous fat thickness $[\mathrm{cm}] \times 40.5278])$. While uncorrected rectus femoris echo intensity values have been reported herein, it should be noted that correction for subcutaneous thickness would not have affected this study's overall conclusions.

\section{Ultrasonography Test-Retest Reliability}


240

241

242

243

244

245

246

247

248

249

250

251

252

253

254

255

256

257

258

259

260

261

262

263

264

265

266

267

268

269

270

271

272

273

274

275

276

277

278

279

Test-retest reliability statistics were calculated for each of the dependent variables using the equations described by Weir (2005). Specifically, we assessed the intraclass correlation coefficient (ICC [model 2, k]), standard error of measurement (SEM [expressed both in absolute terms and as a percentage of the mean]), and the minimal difference needed to be considered real (MD). As presented in a recent publication (Burton and Stock 2018), our laboratory's test-retest reliability statistics for rectus femoris echo intensity in college-aged females were: ICC $=.928$; $\mathrm{SEM}=4.21 \mathrm{AU}(4.22 \%)$; $\mathrm{MD}=11.68 \mathrm{AU}$. For rectus femoris cross-sectional area, these statistics were as follows (Burton and Stock 2018): $\mathrm{ICC}=.982 ; \mathrm{SEM}=0.24 \mathrm{~cm}^{2}(5.43 \%) ; \mathrm{MD}=$ $0.67 \mathrm{~cm}^{2}$. For the vastus lateralis, the echo intensity statistics were: $\mathrm{ICC}=.810 ; \mathrm{SEM}=3.31$ (3.71\%); $\mathrm{MD}=9.17 \mathrm{AU}$. The test-retest reliability statistics for vastus lateralis cross-sectional area were: $\mathrm{ICC}=.980 ; \mathrm{SEM}=0.52(3.43 \%) ; \mathrm{MD}=1.45 \mathrm{~cm}^{2}$.

\section{Actigraphy}

In order to assess compliance with the established protocol, participants were asked to wear Actigraph GT9X accelerometers (ActiGraph Inc, Penscola, FL, USA) on both their right and left legs, fastened securely around each ankle via Velcro straps. The accelerometers detect normal human motion while filtering out high-frequency vibrations that would artificially increase movement data. Participants were instructed to wear accelerometers around both ankles for the entire study period, only removing the devices during showering. Two levels of compliance were established for the current protocol: 1) overall wear-time compliance and 2) compliance to the immobilization protocol. To meet wear-time compliance criteria, established by Troiano (2007), participants were required to wear the device for a minimum of four days (10 hours per day) over a 7-day period. As participants were instructed to wear the device over a two-week period, eight full days of data, including two weekend days, were required to meet compliance criteria. To determine compliance to the immobilization protocol, criteria was established based on a previous study by Cook et al. (2006), who examined differences in both the number of steps and intensity of steps between legs in non-weight bearing participants on crutches.

\section{Statistical Analyses}

All variables were first checked for normal distributions with Shapiro-Wilk tests. To assess compliance with protocol, paired samples t-tests were conducted to determine differences in activity intensity and steps between the immobilized and control limbs of each participant. Two-way (time [pre, post] $\times$ limb [immobilized, control]) repeated measures analyses of variance (ANOVAs) were used to examine mean differences for each muscle and dependent variable. When appropriate, follow-up analyses included Bonferroni post-hoc comparisons and paired samples $t$-tests. We also evaluated $95 \%$ confidence intervals for mean differences (CIs) and effect sizes via Cohen's $d$ statistics. Small, medium, and large Cohen's d corresponded to values of 0.20, 0.50, and 0.80, respectively (Cohen 1988). All statistical procedures were carried out with JASP software (version 0.8.3.1, University of Amsterdam, Amsterdam, The Netherlands). 


\section{Results}

281 Mean \pm SD values for all ultrasonography variables have been displayed in Table 1 .

282 [TABLE 1 ABOUT HERE]

283 Vastus Lateralis Echo Intensity

284

285

286

287

288

289

290

291

292

293

294

295

296

297

298

299

300

301

302

303

304

305

306

307

308

309

310

311

312

313

314

315

316

317

318

319

The results from the two-way repeated measures ANOVA indicated that there was a limb $\times$ time interaction $(F=9.14, p=.011$ [Figure 2]). Follow-up analyses revealed a significant increase in echo intensity $(p=.006,95 \% \mathrm{CI}=-5.60--1.16 \mathrm{AU})$ for the immobilized limb that was considered large (mean increase $=3.40 \mathrm{AU}, d=.918$ ). In contrast, there was a small decrease in echo intensity for the control limb that was not significant (mean decrease $=1.87$ $\mathrm{AU}, p=.189, d=.386,95 \% \mathrm{CI}=-1.06$ to $4.79 \mathrm{AU}$ ). Differences between limbs were small/moderate but not significant at the pretest $(p=.064, d=.567,95 \% \mathrm{CI}=-0.22-6.94 \mathrm{AU})$ or posttest ( $p=.169, d=.406,95 \% \mathrm{CI}=-0.93-4.74 \mathrm{AU})$. For the immobilized limb, three participants showed an increase in vastus lateralis echo intensity that exceeded the MD (9.17 AU). The mean increase in echo intensity for the immobilized limb (3.40 AU) did not exceed the MD.

\section{Vastus Lateralis Cross-Sectional Area}

Despite a large effect size, the results from the two-way repeated measures ANOVA indicated that there was no $\operatorname{limb} \times$ time interaction $(F=3.11, p=.103$ [Figure 3]) and no main effects for limb $(F=1.81, p=.204)$ or time $(F=2.75, p=.123)$. Analysis of effect sizes and 95\% CIs for mean differences revealed similar cross-sectional area values for the pretest and posttest for the control limb $\left(d=.151,95 \% \mathrm{CI}=-0.64-1.08 \mathrm{~cm}^{2}\right)$. However, a moderate effect size was found for the immobilized limb $\left(d=.570,95 \% \mathrm{CI}=-0.07-2.28 \mathrm{~cm}^{2}\right)$. For the immobilized limb, five participants showed a decrease in vastus lateralis cross-sectional area that exceeded the MD $\left(1.40 \mathrm{~cm}^{2}\right)$. The mean decrease in cross-sectional area for the immobilized limb $\left(1.11 \mathrm{~cm}^{2}\right)$ did not exceed the MD.

\section{Correlation between Changes in Vastus Lateralis Echo Intensity and Cross- Sectional Area}

The correlation between change scores for vastus lateralis echo intensity and crosssectional area of the immobilized limb was statistically significant $(r=-.649, p=.016$ [Figure 4]).

\section{[FIGURES 2-4 ABOUT HERE]}

\section{Rectus Femoris Echo Intensity}

The results from the two-way repeated measures ANOVA indicated that there was no limb $\times$ time interaction $(F=.308, p=.589)$ and no main effects for limb $(F=.046, p=.833)$ or time $(F=.004, p=.953$ [Figure 2]). Analysis of effect sizes and $95 \%$ CIs for mean differences revealed nearly identical echo intensity values for the pretest and posttest for the control $(d=$ $.087,95 \% \mathrm{CI}=-3.34-4.47 \mathrm{AU})$ and immobilized $(d=.084,95 \% \mathrm{CI}=-3.32-2.51 \mathrm{AU})$ limbs. For the immobilized limb, none of the participants showed an increase in rectus femoris echo intensity that exceeded the MD (11.68 AU). The mean increase in echo intensity for the immobilized limb (0.41 AU) did not exceed the MD. 
320

321

322

323

324

325

326

327

328

329

330

331

332

333

334

335

336

337

338

339

340

341

342

343

344

345

346

347

348

349

350

351

352

353

354

355

356

357

358

359

\section{Rectus Femoris Cross-Sectional Area}

The results from the two-way repeated measures ANOVA indicated that there was no limb $\times$ time interaction $(F=.041, p=.794)$ and no main effects for limb $(F=.067, p=.801)$ or time $(F=.450, p=.515$ [Figure 3]). Analysis of effect sizes and $95 \%$ CIs for mean differences revealed similar cross-sectional area values for the pretest and posttest for the control $(d=.086$, $\left.95 \% \mathrm{CI}=-0.41-0.55 \mathrm{~cm}^{2}\right)$ and immobilized $\left(d=.309,95 \% \mathrm{CI}=-0.21-0.65 \mathrm{~cm}^{2}\right)$ limbs. For the immobilized limb, four participants showed a decrease in rectus femoris cross-sectional area that exceeded the MD $\left(0.67 \mathrm{~cm}^{2}\right)$. The mean decrease in cross-sectional area for the immobilized $\operatorname{limb}\left(0.22 \mathrm{~cm}^{2}\right)$ did not exceed the MD.

\section{Correlation between Changes in Rectus Femoris Echo Intensity and Cross- Sectional Area}

The correlation between the change in rectus femoris echo intensity and cross-sectional area of the immobilized limb was not statistically significant $(r=.378, p=.202$ [Figure 4]).

\section{Compliance}

After removal of one participant because of difficulty with image analysis, the results from the wear-time validation software indicated that all but one participant met the minimum wear-time criteria ( 8 days of $10+$ hours), with participants wearing the device for an average of $13.1 \pm 1.3$ days. Additionally, significant differences existed between minutes of vigorous physical activity (mean difference of 70.2 minutes $\pm 52.8 ; p=0.002$ ) and total steps (mean difference of 14,861 steps $\pm 19,535 ; p=0.04$ ) between the immobilized limb and the control limb.

\section{Discussion}

Echo intensity is becoming increasingly utilized by investigators to assess changes in skeletal muscle composition (i.e., muscle quality) during various interventions. The major finding of the present study was that two weeks of left knee joint immobilization resulted in a significant increase in echo intensity for the vastus lateralis muscle, and this change was slightly greater than that for the decrease in cross-sectional area. No changes were found for the rectus femoris muscle, which is consistent with previous findings (Hackney and Ploutz-Snyder 2012) and its continued role as a hip flexor throughout the two week intervention. Overall, these results suggest that echo intensity, which appears to reflect intramuscular adiposity (Reimers et al. 1993; Young et al. 2015) and fibrous tissue infiltration (Arts et al. 2012; Pillen et al. 2009), may be a more sensitive measure of muscle morphology than cross-sectional area when studying limb disuse. It is important to note, however, that the magnitude of these changes varied across participants, as many of the pretest-posttest differences did not exceed our laboratory's MD statistics.

The results of the present study supported the hypothesis that there would be greater changes in echo intensity compared to cross-sectional area. Although our study design did not include ultrasonography testing at multiple time points, these findings suggest that the deterioration in muscle quality may occur early in the disuse period and - perhaps more 
360

361

362

363

364

365

366

367

368

369

370

371

372

373

374

375

376

377

378

379

380

381

382

383

384

385

386

387

388

389

390

391

392

393

394

395

396

397

398

399

importantly - prior to the onset of notable atrophy. To our knowledge, our two week study is the first to find potential indications of adipose tissue changes following as little as two week of disuse. To our understanding, only two previous studies have investigated infiltration of intramuscular adipose tissue following a period of disuse (Gorgey and Dudley 2007; Manini et al. 2007). Manini et al. (2007) found a $14.5 \%$ increase in intermuscular adipose tissue in the thigh musculature following a four-week period of unilateral lower limb suspension. Of interesting note, this accumulation of intermuscular fat occurred prior to any significant changes in subcutaneous fat content. The hypothesized physiological explanation for the increased intermuscular fat content was due to a suppression of skeletal muscle lipase activity during periods of inactivity. Similar findings were found in participants who had been diagnosed with an acute, incomplete spinal cord injury (Gorgey and Dudley 2007). The disuse of the lower extremities occurred in a more clinical manner, in which the participants were primarily wheelchair dependent due to the nature of the injury. Substantial increases in intramuscular adipose tissue were found in the thigh musculature six weeks after the injury. The largest and most dramatic increase was seen during the initial six weeks, but there was continued infiltration of intramuscular fat at three months follow up (Gorgey and Dudley 2007). While other studies specifically addressing changes in muscle quality following disuse are limited, insight into this topic may be gleamed from the aging literature, as aging and inactivity seem to manifest in similar physiological adaptations (Bell et al. 2016). Specifically, high echo intensity values have been associated with muscle weakness in the elderly population (Watanabe et al. 2013). Muscle quality in older adults has also been shown to be a significant predictor of performance during the 30-second chair stand test (Lopez et al. 2017; Rech et al. 2014), which has important functional relevance. Given that periods of disuse are common in many medical scenarios, such as ACL tears, our findings highlight the need for researchers and health care professionals to focus their attention on deterioration of muscle quality, rather than just atrophy. A post-operative ACL reconstruction patient, for whom weight bearing is contraindicated, may suffer a reduction in muscle quality despite no noticeable change in muscle size. These results suggest that further research is warranted to investigate early interventions to mitigate the effects of short-term disuse on muscle quality.

The moderate decline in cross-sectional area observed in this study is in line with a variety of previous studies. Following a comprehensive review of unilateral limb immobilization studies, Hackney and Ploutz-Snyder (2012) determined that the vastus lateralis was significantly more prone to disuse than the gastrocnemius and the rectus femoris. Based on the available literature available at the time, it was estimated that the vastus lateralis atrophies at a rate of $.44 \%$ per day. Our design and results seem to be quite similar to the work of Wall et al. (2014), who also studied a two-week duration. These authors reported significant atrophy of the vastus lateralis after only five days of immobilization, demonstrating a 3.5\% decrease. The decline in cross-sectional area continued as the period of immobilization prolonged, reaching a $8.4 \%$ decrease by 14 days. It should be noted that our focus was on healthy females, primarily due the relevance to knee joint injuries in athletics (Prodromos et al. 2007; Renstrom et al. 2008). 
400

401

402

403

404

405

406

407

408

409

410

411

412

413

414

415

416

417

418

419

420

421

422

423

424

425

426

427

428

429

430

431

432

433

434

435

436

437

438

439

Additional studies are needed to investigate if there are differences between sexes and other muscle groups in the ability to preserve muscle quality versus size during a period of disuse. Given that the decline in muscle size was lesser in magnitude than that observed for muscle quality, it is evident that declines in muscle function during periods of immobility are mechanistically related to a variety of physiological phenomena.

To the best of our knowledge, this is the first study to utilize ultrasonography to track changes in echo intensity following limb immobilization. Previous immobilization studies have primarily utilized MRI (Berg et al. 1997; Gorgey and Dudley 2007) and CT-scans (Dirks et al. 2014; Wall et al. 2014) to analyze decreases in cross-sectional area of the quadriceps femoris muscles. While our results provide additional support for the use of ultrasonography-based echo intensity as a time- and cost-effective means of studying muscle morphology, we believe that its exact anatomical underpinnings require further investigation. On one hand, there is strong evidence to support the notion that echo intensity is associated with intramuscular adiposity (Arts et al. 2012; Young et al. 2015). A study by Young et al. (2015) conducted MRI and ultrasonography imaging on 31 participants of varying ages for the rectus femoris, biceps femoris, tibialis anterior, and medial gastrocnemius. They found that there were strong correlations between the percentage of intramuscular fat as shown with MRI and echo intensity as measured with ultrasonography, with the rectus femoris association being particularly high ( $\mathrm{r}$ $=.91$ ). On the other hand, the influence of fibrous tissue content should not be completely discounted. Pillen et al. (2009) analyzed both echo intensity and muscle biopsies of golden retrievers suffering from muscular dystrophy. The results of their study revealed that muscle echo intensity was strongly correlated $(r=.87)$ with the amount of intramuscular fibrous tissue. In addition, the fact that echo intensity is altered by muscle tissue damage following eccentric exercise (Nosaka and Sakamoto 2001) and potentially reflective of glycogen/hydration status (Jenkins 2016) points to the need for more comprehensive studies in this area. Collectively, while the term muscle quality is likely an appropriate term for ultrasonography-based echo intensity, the precise explanation for the changes observed in the present study is worthy of additional inquiry.

Like all studies, this investigation had several limitations that are worthy of brief discussion. First, although our efforts to monitor and ensure compliance give us confidence in these findings, it seems impossible to state with $100 \%$ certainty that our participants did not ever weight bear. Future investigators wishing to perform similar studies in college-aged participants should consider the use of a golf cart to provide transportation to and from classes. Unfortunately, this option was not available when the present study began. Second, while we were motivated to perform this study in young females, the external validity to other populations is somewhat limited. Indeed, future studies are needed in young males as well as varying age groups to determine if the enhanced sensitivity of echo intensity versus cross-sectional area is consistent across sexes and age groups. As echo intensity appears to be lower in African Americans (Melvin et al. 2014), racial and ethnic backgrounds of research participants should also be studied. Third, as our study was designed to specifically examine limb immobilization, 
440 these findings should not be extrapolated to other non-weight bearing conditions, and in 441 particular, bedrest. We believe that the ambulatory activities of the control limb should not be 442 discounted, particularly given the influence of cross education training (Carr et al. 2019) and the 443 central nervous system's role in maintaining muscle strength and mass (Clark et al. 2014).

444 Similarly, our implementation of a $90^{\circ}$ joint angle at the knee to ensure that the participants' toes 445 did not come into contact with the ground during ambulating with crutches resulted in a unique 446 biomechanical condition, particularly for the biarticular muscles of the thigh. Finally, it is 447 interesting to note that although some group mean differences and moderate/large effect sizes 448 were demonstrated, only a small portion of the participants showed changes that exceeded the 449 MD. None of the mean differences exceeded the MD. This contrast was despite good-to450 excellent test-retest reliability statistics of well-trained and experienced investigators. These 451 results highlight the need to consider change scores on a participant-by-participant basis, as well 452 as the practical value of including multiple and comprehensive analytical approaches in research 453 (i.e., effect sizes, confidence intervals, $p$ values, individual participant data, etc.).

454

455

456

457

458

459

460

461

462

463

464

465

466

467

468

469

470

471

472

473

474

475

476

477

478

479

480

481

\section{Conclusions}

We sought to examine the effects of two weeks of knee joint immobilization on vastus lateralis and rectus femoris muscle quality and size. The findings of this study demonstrated that muscle quality deteriorated to a greater extent than muscle size during two weeks of knee joint immobilization in college-aged females. We believe that the lack of change for the rectus femoris due to its role as a hip flexor provides further justification for this conclusion. Future investigators may wish to verify these results in males and participants of various age and ethnic groups. Based on these data, ultrasonography-based echo intensity appears to be a useful clinical tool for tracking changes in skeletal muscle deterioration during extended periods of disuse.

\section{References}

Arts IM, Schelhaas HJ, Verrijp KC, Zwarts MJ, Overeem S, van der Laak JA, Lammens MM, Pillen S (2012) Intramuscular fibrous tissue determines muscle echo intensity in amyotrophic lateral sclerosis. Muscle \& Nerve 45:449-450 doi:10.1002/mus.22254

Bell KE, von Allmen MT, Devries MC, Phillips SM (2016) Muscle Disuse as a Pivotal Problem in Sarcopenia-related Muscle Loss and Dysfunction. The Journal of Frailty \& Aging 5:33-41 doi:10.14283/jfa.2016.78

Beck TW (2013) The importance of a priori sample size estimation in strength and conditioning research. The Journal of Strength and Conditioning Research 8:2323-2337 doi: 10.1519/JSC.0b013e318278eea0

Berg HE, Larsson L, Tesch PA (1997) Lower limb skeletal muscle function after 6 wk of bed rest. J Appl Physiol (1985) 82:182-188 doi:10.1152/jappl.1997.82.1.182

Burton AM, Stock MS (2018) Consistency of novel ultrasound equations for estimating percent intramuscular fat. Clinical Physiology and Functional Imaging doi:10.1111/cpf.12532

Campbell M, Varley-Campbell J, Fulford J, Taylor B, Mileva KN, Bowtell JL (2019) Effect of immobilisation on neuromuscular function in vivo in humans: A systematic review. Sports Medicine 49:931-950. doi: 10.1007/s40279-019-01088-8.

Peer] reviewing PDF | (2019:08:40344:2:0:NEW 7 Nov 2019) 
482 Carr JC, Ye X, Stock MS, Bemben MG, DeFreitas JM (2019) The time course of cross-

483

484

485

486

487

488

489

490

491

492

493

494

495

496

497

498

499

500

501

502

503

504

505

506

507

508

509

510

511

512

513

514

515

516

517

518

519

520

521

522

523

524

525

526

527 education during short-term isometric strength training. European Journal of Applied Physiology doi:10.1007/s00421-019-04130-9

Clark BC, Mahato NK, Nakazawa M, Law TD, Thomas JS (2014) The power of the mind: the cortex as a critical determinant of muscle strength/weakness. J Neurophysiol 112:32193226 doi:10.1152/jn.00386.2014

Cohen J (1988) Statistical Power Analysis for the Behavioral Sciences. 2nd edn. Lawrence Erlbaum Associates, Hillsdale, NJ

Cook SB, Clark BC, Ploutz-Snyder LL (2006) Accelerometry as a measure of subject compliance in unilateral lower limb suspension. Aviation, Space, and Environmental Medicine 77:953-956

Deschenes MR, Holdren AN, McCoy RW (2008) Adaptations to short-term muscle unloading in young and aged men. Med Sci Sports Exerc 40:856-863 doi:10.1249/MSS.0b013e318164f4b6

Deschenes MR, McCoy RW, Holdren AN, Eason MK (2009) Gender influences neuromuscular adaptations to muscle unloading. European Journal of Applied Physiology 105:889-897 doi:10.1007/s00421-008-0974-5

Deschenes MR, McCoy RW, Mangis KA (2017) Chronic Resistance Training Does Not Ameliorate Unloading-Induced Decrements in Neuromuscular Function. American Journal of Physical Medicine \& Rehabilitation / Association of Academic Physiatrists 96:549-556 doi:10.1097/PHM.0000000000000681

Dirks ML, Wall BT, Snijders T, Ottenbros CL, Verdijk LB, van Loon LJ (2014) Neuromuscular electrical stimulation prevents muscle disuse atrophy during leg immobilization in humans. Acta Physiologica 210:628-641 doi:10.1111/apha.12200

Fairchild SL (2012) Pierson and Fairchild's Principles \& Techniques of Patient Care. 5th edn. Elsevier Saunders, St. Louis, Missouri

Gorgey AS, Dudley GA (2007) Skeletal muscle atrophy and increased intramuscular fat after incomplete spinal cord injury. Spinal Cord 45:304-309 doi:10.1038/sj.sc.3101968

Hackney KJ, Ploutz-Snyder LL (2012) Unilateral lower limb suspension: integrative physiological knowledge from the past 20 years (1991-2011). European Journal of Applied Physiology 112:9-22 doi:10.1007/s00421-011-1971-7

Hiemstra LA, Heard SM, Sasyniuk TM, Buchko GL, Reed JG, Monteleone BJ (2009) Knee immobilization for pain control after a hamstring tendon anterior cruciate ligament reconstruction: a randomized clinical trial. The American Journal of Sports Medicine 37:56-64 doi:10.1177/0363546508322896

Jenkins ND (2016) Are Resistance Training-Mediated Decreases in Ultrasound Echo Intensity Caused by Changes in Muscle Composition, or Is There an Alternative Explanation? Ultrasound in Medicine \& Biology 42:3050-3051 doi:10.1016/j.ultrasmedbio.2016.07.011

Kleinberg CR, Ryan ED, Tweedell AJ, Barnette TJ, Wagoner CW (2016) Influence of Lower Extremity Muscle Size and Quality on Stair-Climb Performance in Career Firefighters. Journal of Strength and Conditioning Research / National Strength \& Conditioning Association 30:1613-1618 doi:10.1519/JSC.0000000000001268

Lopez P, Wilhelm EN, Rech A, Minozzo F, Radaelli R, Pinto RS (2017) Echo intensity independently predicts functionality in sedentary older men. Muscle \& Nerve 55:9-15 doi:10.1002/mus.25168 
528

529

530

531

532

533

534

535

536

537

538

539

540

541

542

543

544

545

546

547

548

549

550

551

552

553

554

555

556

557

558

559

560

561

562

563

564

565

566

567

568

569

570

571

572

Manini TM, Clark BC, Nalls MA, Goodpaster BH, Ploutz-Snyder LL, Harris TB (2007) Reduced physical activity increases intermuscular adipose tissue in healthy young adults. Am J Clin Nutr 85:377-384 doi:10.1093/ajen/85.2.377

Mather RC 3rd, Koenig L, Kocher MS, Dall TM, Gallo P, Scott DJ, Bach BR Jr, Spindler KP; MOON Knee Group (2013) Societal and economic impact of anterior cruciate ligament tears The Journal of Bone and Joint Surgery-American Volume 95:1751-1759 doi:10.2106/JBJS.L.01705

Melvin MN, Smith-Ryan AE, Wingfield HL, Fultz SN, Roelofs EJ (2014) Evaluation of muscle quality reliability and racial differences in body composition of overweight individuals. Ultrasound in Medicine \& Biology 40:1973-1979 doi:10.1016/j.ultrasmedbio.2014.03.012

Mota JA, Stock MS, Thompson BJ (2017) Vastus lateralis and rectus femoris echo intensity fail to reflect knee extensor specific tension in middle-school boys. Physiological Measurement 38:1529-1541 doi:10.1088/1361-6579/aa791a

Newman AB, Kupelian V, Visser M, Simonsick EM, Goodpaster BH, Kritchevsky SB, Tylavsky FA, Rubin SM, Harris TB (2006) Strength, but not muscle mass, is associated with mortality in the health, aging and body composition study cohort. The Journals of Gerontology Series A, Biological Sciences and Medical Sciences 61:72-77

Nishihara K, Kawai H, Hayashi H, Naruse H, Kimura A, Gomi T, Hoshi F (2014) Frequency analysis of ultrasonic echo intensities of the skeletal muscle in elderly and young individuals. Clinical Interventions in Aging 9:1471-1478.

Nosaka K, Sakamoto K (2001) Effect of elbow joint angle on the magnitude of muscle damage to the elbow flexors. Med Sci Sports Exerc 33:22-29

Pillen S, Tak RO, Zwarts MJ, Lammens MM, Verrijp KN, Arts IM, van der Laak JA, Hoogerbrugge PM, van Engelen BG, Verrips A (2009) Skeletal muscle ultrasound: correlation between fibrous tissue and echo intensity. Ultrasound in Medicine \& Biology 35:443-446 doi:10.1016/j.ultrasmedbio.2008.09.016

Prodromos CC, Han Y, Rogowski J, Joyce B, Shi K (2007) A meta-analysis of the incidence of anterior cruciate ligament tears as a function of gender, sport, and a knee injury-reduction regimen. Arthroscopy: The Journal of Arthroscopic \& Related Surgery: Official publication of the Arthroscopy Association of North America and the International Arthroscopy Association 23:1320-1325 e1326 doi:10.1016/j.arthro.2007.07.003

Rech A, Radaelli R, Goltz FR, da Rosa LH, Schneider CD, Pinto RS (2014) Echo intensity is negatively associated with functional capacity in older women. Age 36:9708 doi:10.1007/s11357-014-9708-2

Reimers K, Reimers CD, Wagner S, Paetzke I, Pongratz DE (1993) Skeletal muscle sonography: a correlative study of echogenicity and morphology. Journal of Ultrasound in Medicine: Official journal of the American Institute of Ultrasound in Medicine 12:73-77

Renstrom P, Ljungqvist A, Arendt E, Beynnon B, Fukubayashi T, Garrett W, Georgoulis T, Hewett TE, Johnson R, Krosshaug T, Mandelbaum B, Micheli L, Myklebust G, Roos E, Roos H, Schamasch P, Shultz S, Werner S, Wojtys E, Engebretsen L (2008) Non-contact ACL injuries in female athletes: An International Olympic Committee current concepts statement. British journal of Sports Medicine 42:394-412 doi:10.1136/bjsm.2008.048934

Sanders TL, Maradit Kremers H, Bryan AJ, Larson DR, Dahm DL, Levy BA, Stuart MJ, Krych AJ (2016) Incidence of Anterior Cruciate Ligament Tears and Reconstruction: A 21-Year

Peer) reviewing PDF | (2019:08:40344:2:0:NEW 7 Nov 2019) 
573

574

575

576

577

578

579

580

581

582

583

584

585

586

587

588

589

590

591

592

593

594

595

596

597

598

599

600

601
Population-Based Study. The American Journal of Sports Medicine 44:1502-1507 doi:10.1177/0363546516629944

Schulze K, Gallagher P, Trappe S (2002) Resistance training preserves skeletal muscle function during unloading in humans. Med Sci Sports Exerc 34:303-313

Stevens JP (2007) Intermediate Statistics: A Modern Approach. 3rd edn. Lawrence Erlbaum Associates; Taylor \& Francis Group, New York, NY

Stock MS, Mota JA, Hernandez JM, Thompson BJ (2017) Echo intensity and muscle thickness as predictors Of athleticism and isometric strength in middle-school boys. Muscle \& Nerve 55:685-692 doi:10.1002/mus.25395

Tesch PA, Trieschmann JT, Ekberg A (2004) Hypertrophy of chronically unloaded muscle subjected to resistance exercise. J Appl Physiol (1985) 96:1451-1458 doi:10.1152/japplphysiol.01051.2003

Troiano RP (2007) Large-scale applications of accelerometers: new frontiers and new questions. Med Sci Sports Exerc 39:1501 doi:10.1097/mss.0b013e318150d42e

Vinogradova Y, Coupland C, Hippisley-Cox J (2015) Use of combined oral contraceptives and risk of venous thromboembolism: nested case-control studies using the QResearch and CPRD databases. BMJ 350:h2135 doi:10.1136/bmj.h2135

Wall BT, Dirks ML, Snijders T, Senden JM, Dolmans J, van Loon LJ (2014) Substantial skeletal muscle loss occurs during only 5 days of disuse. Acta Physiologica 210:600-611 doi:10.1111/apha.12190

Watanabe Y, Yamada Y, Fukumoto Y, Ishihara T, Yokoyama K, Yoshida T, Miyake M, Yamagata E, Kimura M (2013) Echo intensity obtained from ultrasonography images reflecting muscle strength in elderly men. Clinical Interventions in Aging 8:993-998 doi:10.2147/CIA.S47263

Weir JP (2005) Quantifying test-retest reliability using the intraclass correlation coefficient and the SEM. Journal of Strength and Conditioning Research / National Strength \& Conditioning Association 19:231-240 doi:10.1519/15184.1

Young HJ, Jenkins NT, Zhao Q, McCully KK (2015) Measurement of intramuscular fat by muscle echo intensity. Muscle \& Nerve 52:963-971 doi:10.1002/mus.24656 


\section{Table $\mathbf{1}$ (on next page)}

Two weeks of knee joint immoblization result in significant increases in vastus lateralis echo intensity.

Mean +/- SD echo intensity and cross-sectional area values for each muscle and testing session of the immoblized (A.) and control (B.) limbs. ${ }^{*}=p<.05$ 
2

\begin{tabular}{|c|c|c|c|}
\hline \multicolumn{2}{|l|}{ A. } & \multicolumn{2}{|c|}{ Immobilized Limb } \\
\hline \multicolumn{2}{|c|}{ Vastus lateralis cross-sectional area $\left(\mathrm{cm}^{2}\right)$} & \multicolumn{2}{|c|}{ Rectus femoris cross-sectional area $\left(\mathrm{cm}^{2}\right)$} \\
\hline PRE & POST & PRE & POST \\
\hline $16.71 \pm 2.85$ & $15.60 \pm 2.38$ & $6.15 \pm 1.17$ & $5.93 \pm 0.86$ \\
\hline \multicolumn{2}{|c|}{ Vastus lateralis echo intensity (AU) } & \multicolumn{2}{|c|}{ Rectus femoris echo intensity (AU) } \\
\hline PRE & POST & PRE & POST \\
\hline $82.77 \pm 8.35$ & $86.17 \pm 9.54 *$ & $82.85 \pm 7.61$ & $83.25 \pm 10.30$ \\
\hline B. & \multicolumn{2}{|c|}{ Control Limb } & \\
\hline \multicolumn{2}{|c|}{ Vastus lateralis cross-sectional area $\left(\mathrm{cm}^{2}\right)$} & \multicolumn{2}{|c|}{ Rectus femoris cross-sectional area $\left(\mathrm{cm}^{2}\right)$} \\
\hline PRE & POST & PRE & POST \\
\hline $17.05 \pm 3.09$ & $16.84 \pm 3.59$ & $6.17 \pm 1.44$ & $5.95 \pm 1.42$ \\
\hline \multicolumn{2}{|c|}{ Vastus lateralis echo intensity (AU) } & \multicolumn{2}{|c|}{ Rectus femoris echo intensity (AU) } \\
\hline PRE & POST & PRE & POST \\
\hline $86.13 \pm 10.47$ & $84.26 \pm 9.25$ & $83.95 \pm 10.90$ & $83.38 \pm 12.21$ \\
\hline
\end{tabular}




\section{Figure 1}

Use of crutches during knee joint immoblization

An example of a participant being instructed on proper use of crutches in navigating the community.

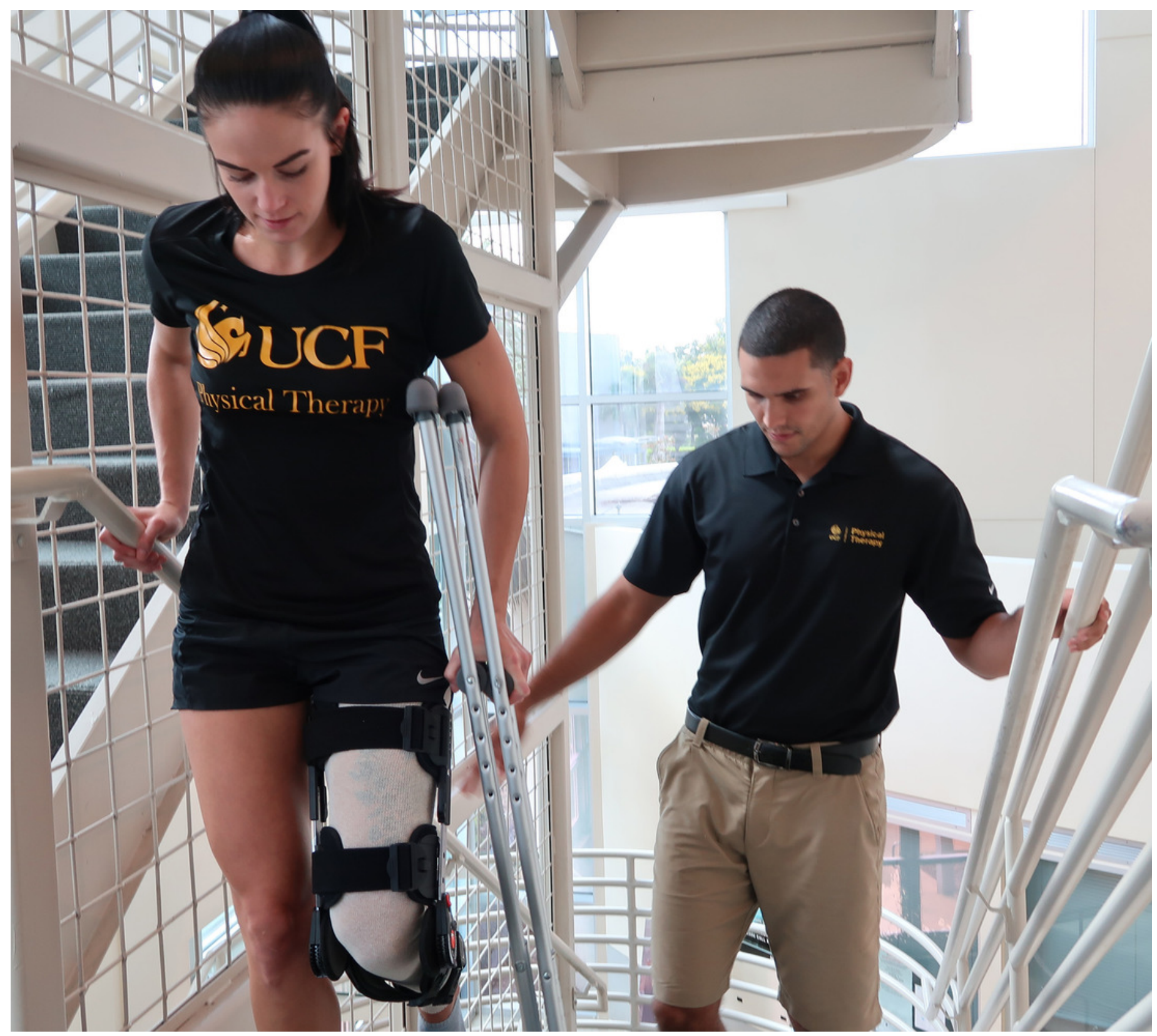


Figure 2

Changes in Muscle Quality During Limb Immobilization

Individual participant data showing changes in echo intensity for the vastus lateralis (A.) and rectus femoris (B.) of the immobilized limb. The thick, dotted lines correspond to the mean values. The mean +/- SD values are displayed for each muscle as well. 
A

\section{Vastus Lateralis; Immobilized Limb}

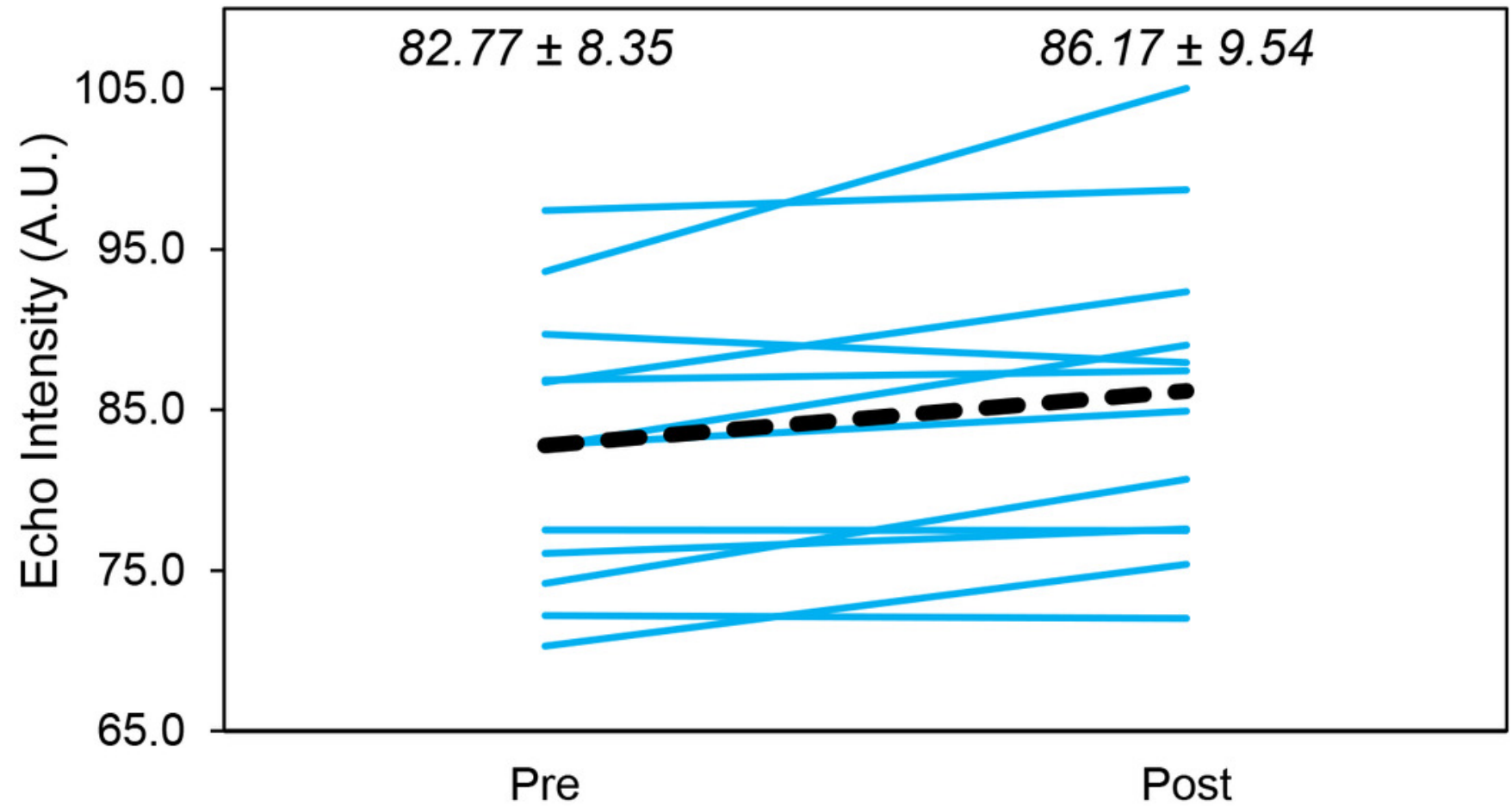

B.

Rectus Femoris; Immobilized Limb

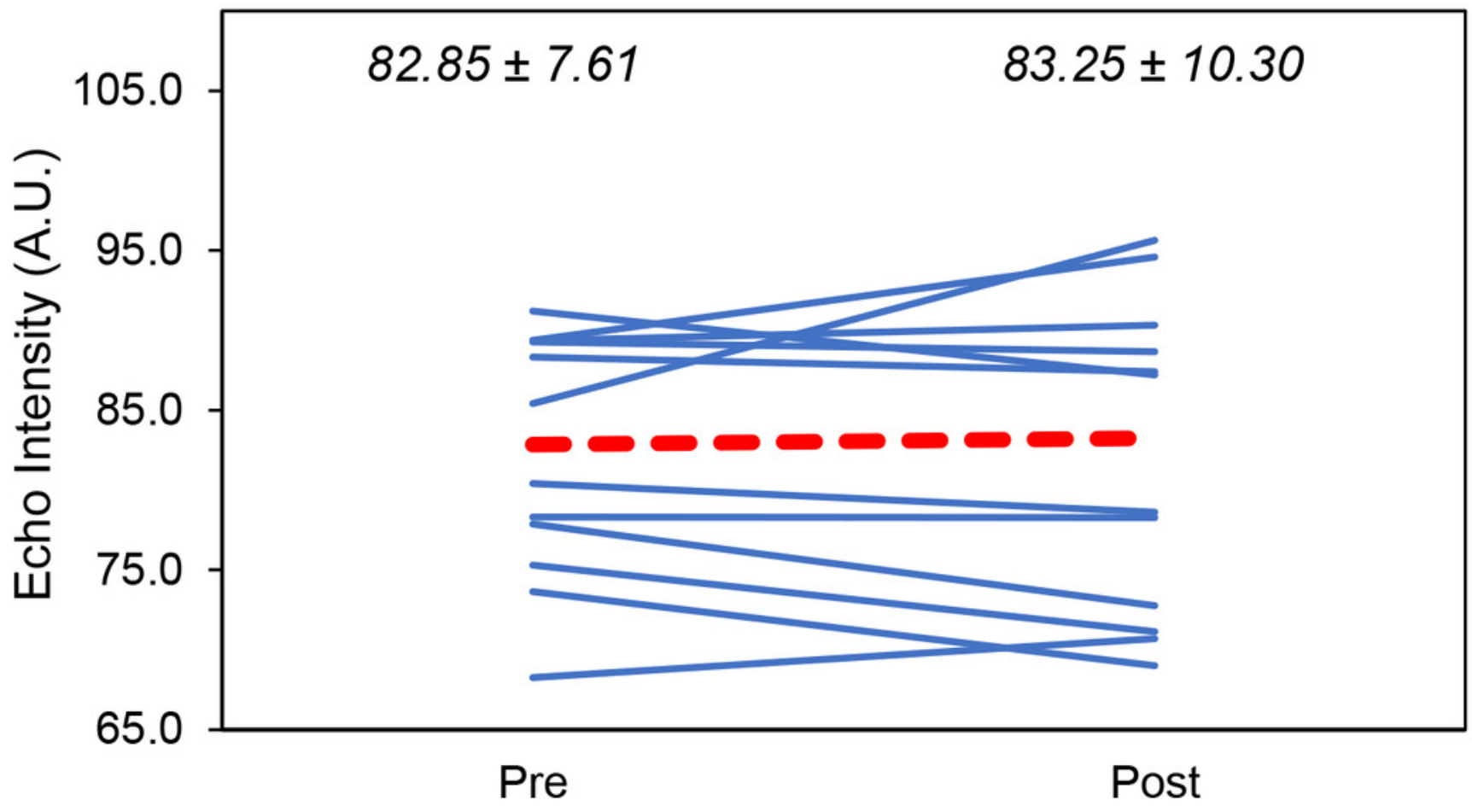




\section{Figure 3}

Changes in Muscle Size During Limb Immobilization

Individual participant data showing changes in cross-sectional area for the vastus lateralis (A.) and rectus femoris (B.) of the immobilized limb. The thick, dotted lines correspond to the mean values. The mean $+/$ - SD values are displayed for each muscle as well. 


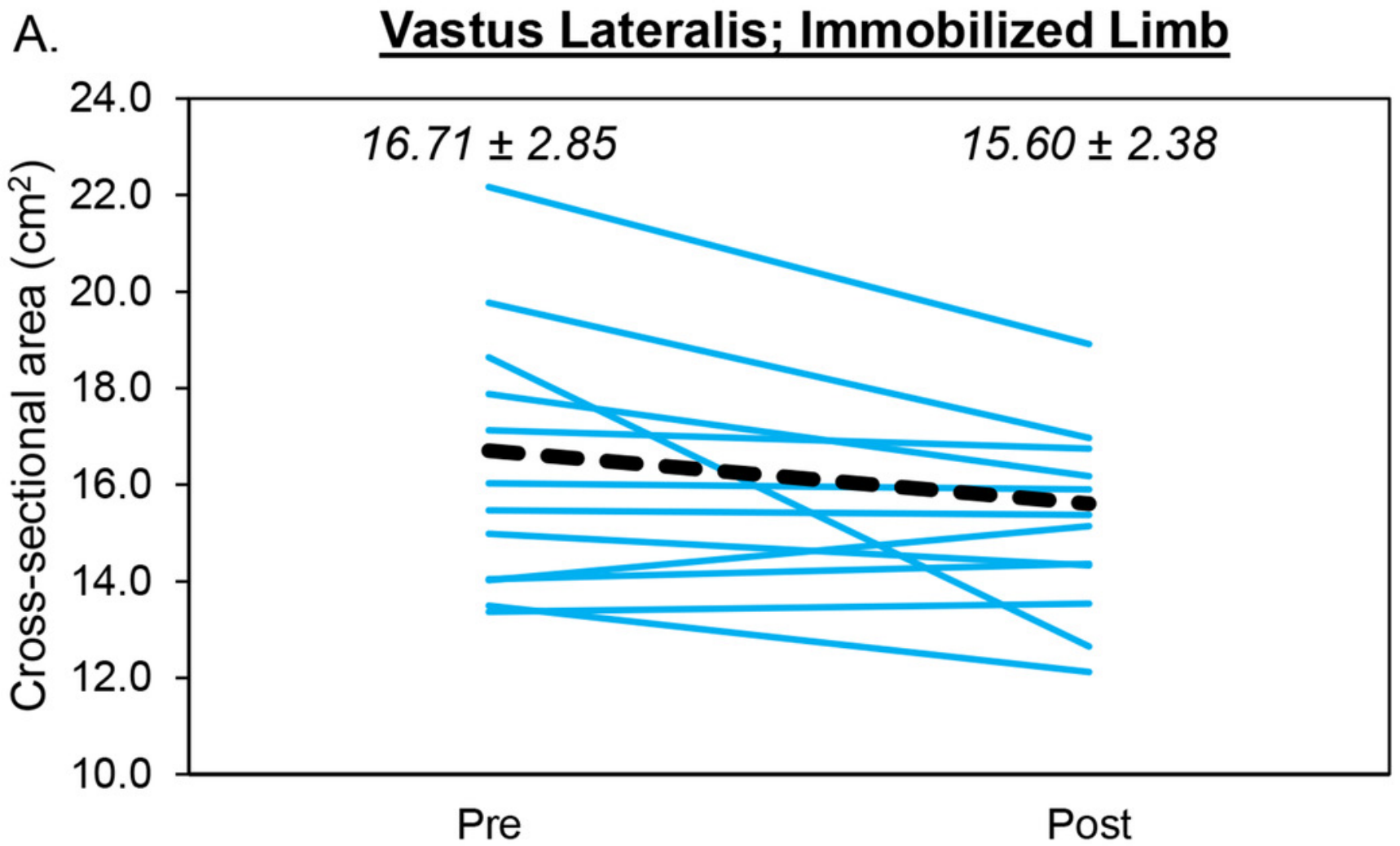

B.

Rectus Femoris; Immobilized Limb

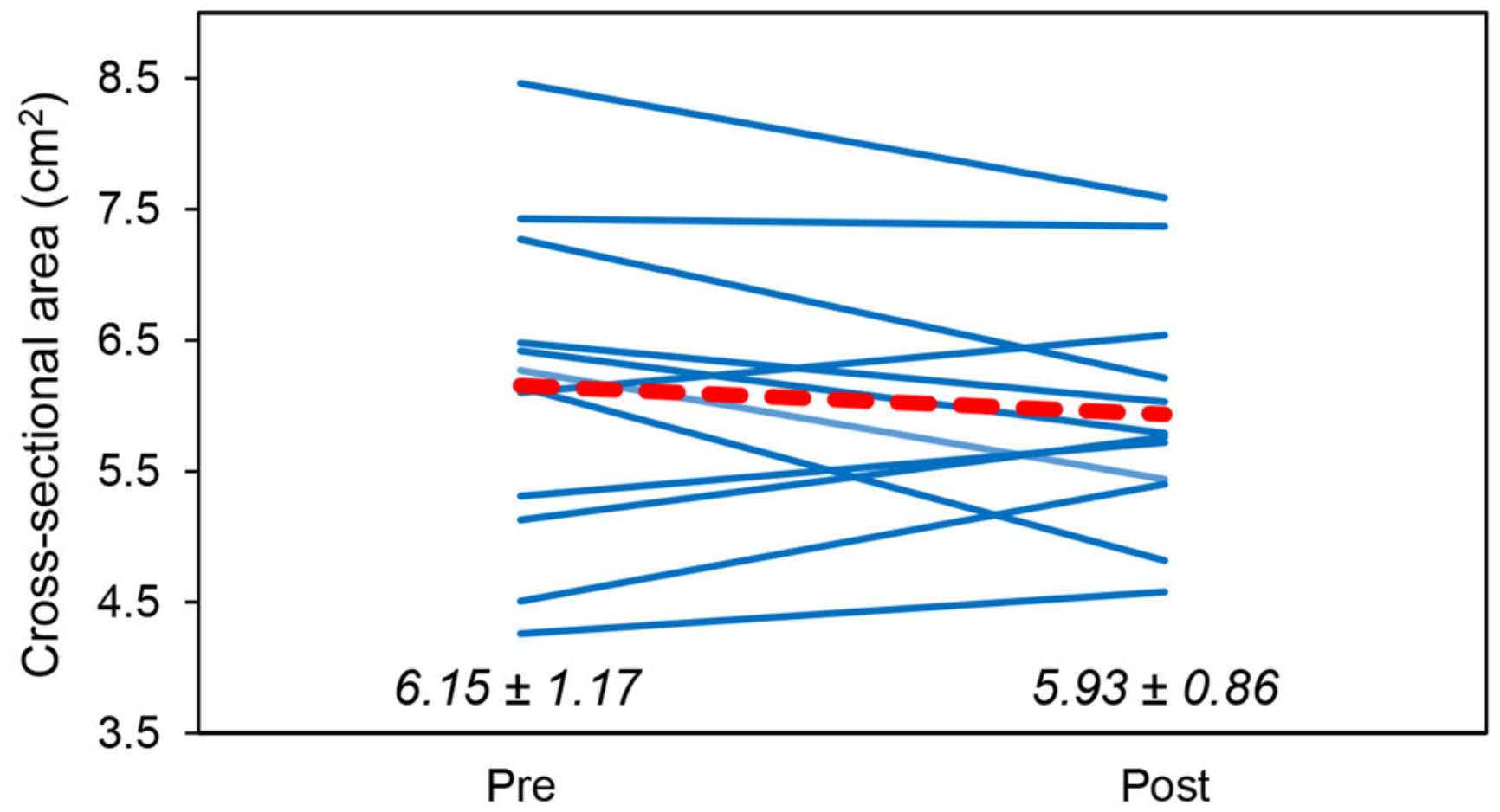


Figure 4

Significant Associations Between Changes in Muscle Quality and Size

Scatterplots displays the statistically significant correlations between changes in muscle cross-sectional area (CSA) and echo intensity (EI) for the vastus lateralis (A.) and rectus femoris (B.). It is important for the reader to keep in mind that changes in rectus femoris CSA and El were small and likely not meaningful. 
A.

\section{Vastus Lateralis}

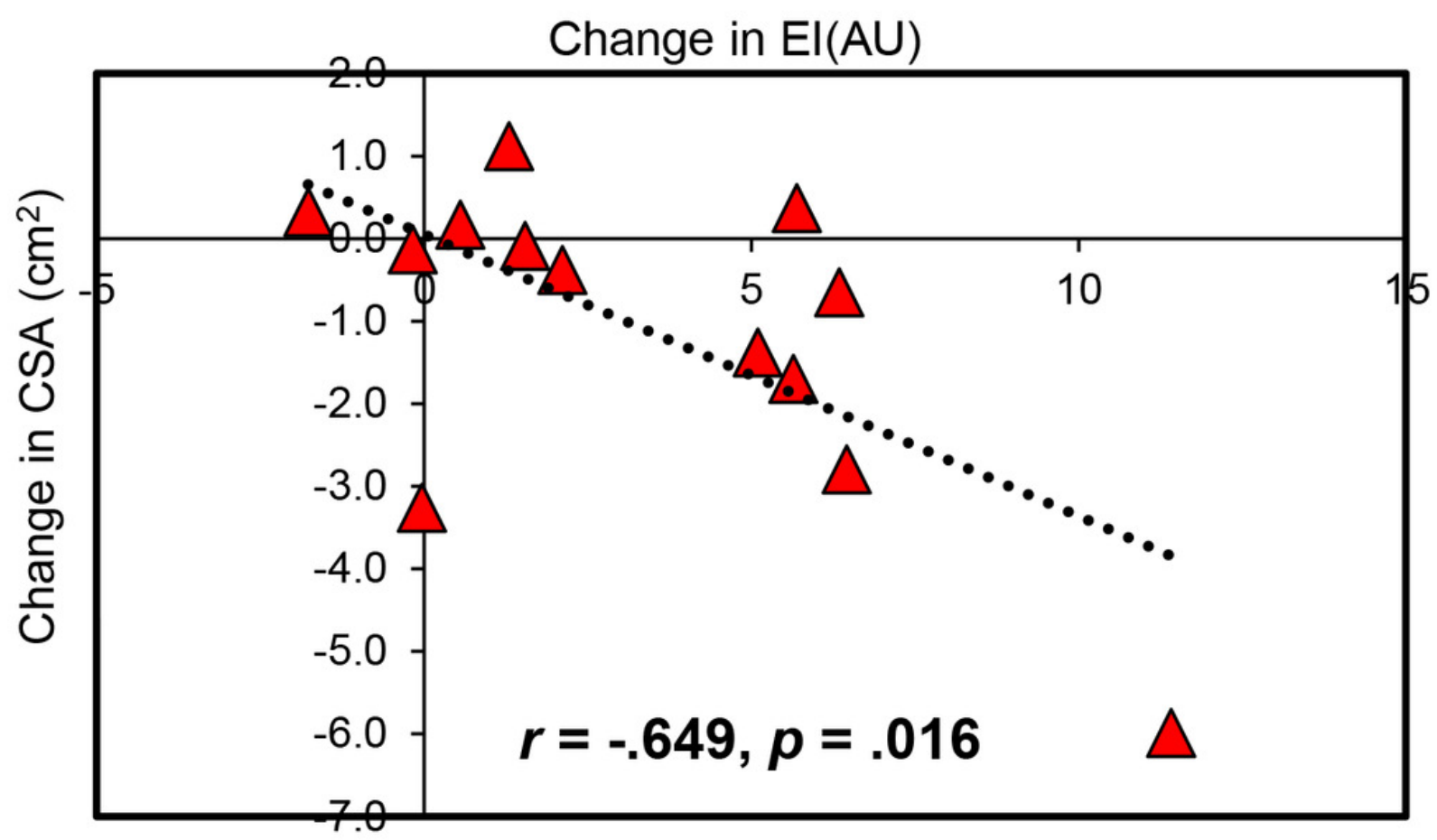

Rectus Femoris

B.

Change in $\mathrm{EI}(\mathrm{AU})$

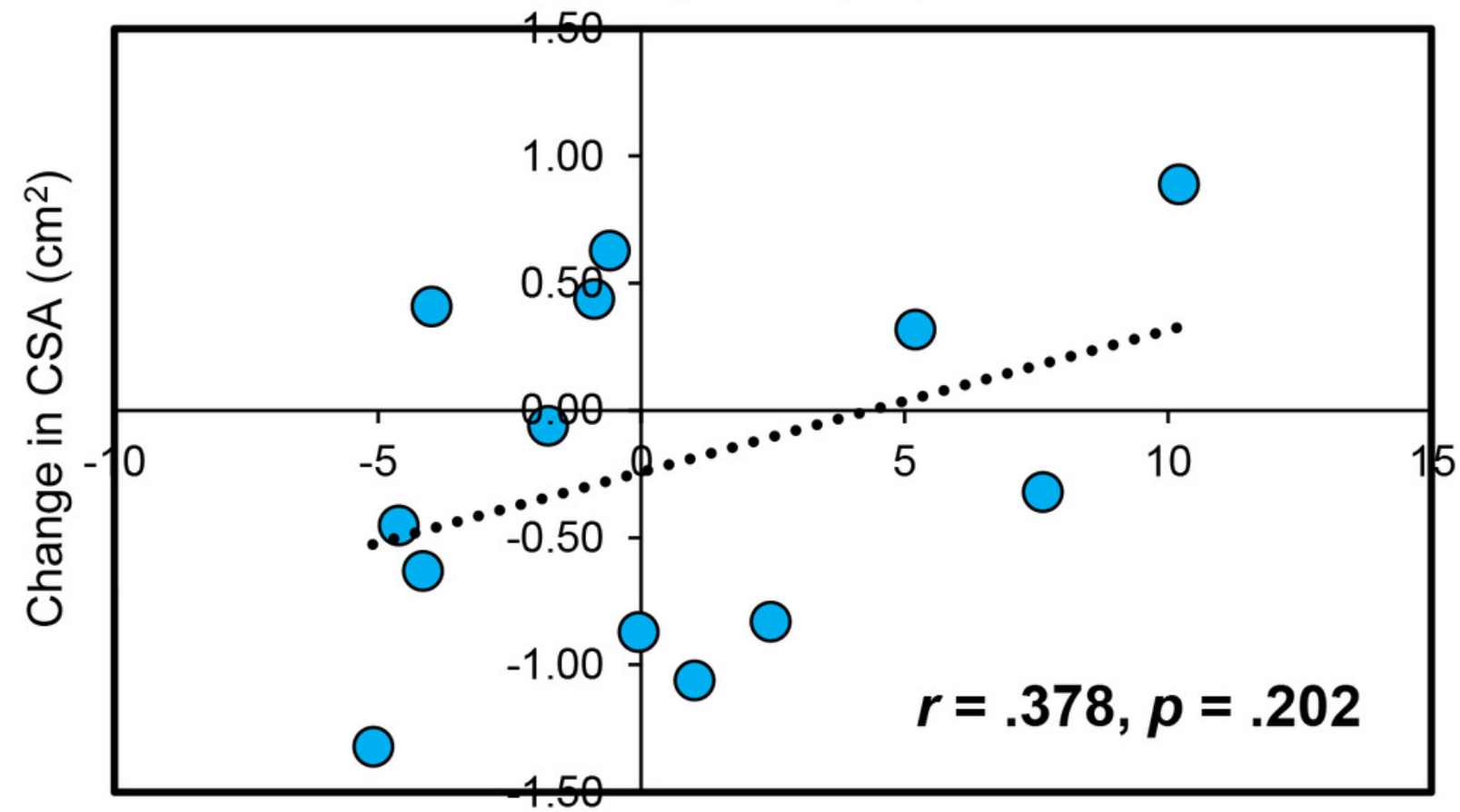

